070.41:811.131.1

929:070.41 Bontempelli M.

https://doi.org/10.18485/italbg.2018.1.4

Sandra Milanko*

Università di Zara

\title{
L'INTERVENTISMO INTELLETTUALE DI MASSIMO BONTEMPELLI TRA GIORNALISMO E BELLICISMO FUTURISTA
}

\begin{abstract}
Questo articolo intende illustrare lo sviluppo e le caratteristiche dell'interventismo di Massimo Bontempelli ravvisabile nella sua attività giornalistica con un accento particolare sui possibili punti di incontro e divergenze con l'interventismo di matrice futurista. I suoi articoli interventisti inseriti nella raccolta Meditazioni intorno alla guerra d'Italia e d'Europa (1917) dimostrano, infatti, che l'interventismo bontempelliano è conducibile all'interventismo democratico piuttosto a quello nazionalista ed imperialista, segnato dalla rievocazione dei valori risorgimentali, dall'uso politico della figura di Garibaldi e dalla retorica antigermanica. Nonostante le iniziali divergenze ideologiche con il discorso interventista del futurismo, sarà proprio la campagna interventista a far avvicinare Bontempelli al movimento di Marinetti prima al livello politico e solo successivamente a quello artistico.

Parole chiave: Bontempelli, interventismo, giornalismo, Grande guerra, futurismo, bellicismo.
\end{abstract}

Come ebbe a rimarcare in diverse occasioni nel corso della sua lunga attività letteraria e giornalistica, Massimo Bontempelli assunse una posizione interventista subito dopo lo scoppio della prima guerra mondiale (Bontempelli 1928). Similmente alla stragrande maggioranza degli artisti e intellettuali nati tra gli anni Settanta e Ottanta del diciannovesimo secolo ${ }^{1}$, il futuro fondatore del realismo magico italiano sostenne la causa interventista pubblicando una fitta serie di articoli tra il 1914 e il 1915. Dopo l'entrata

*smilanko@unizd.hr

${ }^{1}$ Per farsi un quadro generale di coetanei di Bontempelli, nato nel 1878, possiamo individuare alcuni protagonisti dell'Italia pre- o postbellica come Filippo Tommaso Marinetti (1876), Ardengo Soffici (1879), Giovanni Papini (1881), Carlo Carrà (1881), Giuseppe Prezzolini (1882), Renato Serra (1884), Piero Jahier (1884), Aldo Palazzeschi (1885), Giovanni Boine (1887), Giuseppe Ungaretti (1888). 
in guerra dell'Italia, agli articoli faranno seguito delle corrispondenze di guerra che l'autore comasco redigerà da inviato speciale per il periodico «Messaggero». La rilevanza di questi scritti giornalistici verrà conclamata da due raccolte in volumi, pubblicate a distanza di due anni: Dallo Stelvio al mare (1915) e Meditazioni intorno alla guerra d'Italia e d'Europa (1917). Ignorate dalla maggior parte della critica bontempelliana, queste antologie di articoli rivelano non solo le sue posizioni politiche in quanto dichiarato interventista, ma anche l'inizio di quello che Fernando Tempesti (1974) definì una "dialettica non dichiarata" tra Bontempelli e il movimento futurista e che si protrarrà ben oltre la Grande guerra. Infatti, ciò che emerge da questi scritti (in modo particolare dalle corrispondenze dal fronte) è che il suo graduale avvicinamento al futurismo fu dovuto inizialmente (e maggiormente) alle posizioni politiche che i seguaci di Marinetti proclamavano nei primi mesi della guerra ${ }^{2}$. In questa sede, trattando la tipologia dell'interventismo di Bontempelli, ci soffermeremo sulla raccolta del 1917 che include alcuni degli articoli scritti e pubblicati nei mesi della campagna interventista.

Con la legatura in pelle lavorata con fregi in bassorilievo, il volume Meditazioni intorno alla guerra d'Italia e d'Europa è anche editorialmente prestigioso in quanto pubblicato dall'Istituto Editoriale Italiano di Umberto Notari. Questi era uno scrittore, editore e organizzatore culturale nella Milano prebellica. Simpatizzante dell'impresa libica, dell'interventismo e dello stesso futurismo, Notari indubbiamente funge da tramite tra Bontempelli e gli esponenti del movimento futurista. Bontempelli, infatti, prima di trasferirsi in modo definitivo da Firenze a Milano, collabora alle attività culturali dell' Istituto Editoriale Italiano. Le stesse Meditazioni saranno inserite nell'ampia collana Raccolta di breviari italiani, insieme ai quattro volumi di manifesti del futurismo a cura di Marinetti. A differenza della raccolta del 1915 che assume le funzioni di un diario di guerra, gli articoli delle Meditazioni non sono ordinati cronologicamente e comprendono interventi scritti in un arco di tempo più ampio, permettendoci così di esaminare le riflessioni di Bontempelli tra l'agosto del 1914 e il gennaio del 1916.

La raccolta si apre con l'articolo Smarrimento (agosto 1914) in cui Bontempelli proclama subito il suo schieramento interventista. Rivelando nello stesso tempo l'incertezza, l'opportunismo, e persino l'incauto bellicismo di alcuni suoi connazionali, Bontempelli (1917: 16-20) scrive:

\footnotetext{
${ }^{2}$ Una simile ipotesi è altrettanto valida non solo per il breve sodalizio tra «Lacerba» di Papini e Prezzolini e il movimento futurista ma anche per il successivo avvicinamento a Marinetti dei futuri membri della «pattuglia azzurra», Bruno Corra, Arnaldo Ginna ed Emilio Settimelli. Cfr. Papini (1977: 47).
} 
Ora, in quei discorsi, in molti, e in quell'affaccendarsi di tutti, l'intimo e inconscio sentimento di alcuni era questo:

- Io vivo un momento solenne. Io sono spettatore di un'epoca di cui si parlerà da generazioni e generazioni e generazioni di nipoti. Spettatore, cioè parte. Ma che cosa faccio io per essere realmente parte di questo? Leggo, ascolto, commento; poi ripeto quello che ho detto e che ho sentito. Può bastare? Potrà per questo, tra cent'anni, un nipote dire di me: "Fu del tempo della Guerra d'Europa" e forse invidiarmi?

C'era anche taluno che, in un ardore d'azione, rimpiangeva che l'Italia non combattesse. Altri deplorava che l'età, le condizioni civili e le leggi dello stato fossero per impedirgli, anche quando l'Italia prenderà le armi, di aver parte alla guerra $\mathrm{e}$ porsi e sentirsi nel bel mezzo della storia. [...]

Un giovane saggio vagheggiava così:

"Se dovrò andare in guerra, spero che subito, al primo giorno, mi tocchi una ferita in una gamba, da stare in ospedale per qualche mese, fino a guerra finita. Così, con il minimo di perdita, avrò servito la patria ugualmente".

E avrebbe potuto aggiungere: "E sarò ugualmente nella storia". Ma la sua saggezza mi ha fatto fuggire, per pena e per ribrezzo.

L'autore è particolarmente deluso dall'ultimo ragionamento che mostra vigliaccheria, mancanza di senso del dovere, e di comunione; quest'ultimo aspetto cercato inutilmente in mezzo alla natura. Lo smarrimento della nazione allo scoppio della prima guerra mondiale si riversa nello smarrimento dello stesso autore che da questo momento sposa la causa interventista. Così, nell'articolo seguente, Per i poveri letterati (settembre 1914), Bontempelli non solo espone in nuce le idee principali che determineranno la sua futura produzione letteraria ${ }^{3}$, ma invita «i poveri letterati», falliti nel loro mestiere, a rinnovarsi, e a farlo sia attraverso la lettura dei giornali sia andando in guerra $^{4}$. Che Bontempelli abbia trovato un senso di comunione almeno nello schieramento interventista risulta evidente nello scritto Momenti e motivi: $V I$, dello stesso mese, in cui si fa portavoce degli interventisti (Bontempelli 1917: 95-196):

Oggi noi ci sentiamo intorno un'aura d'incertezza, di timidità, di perplessità, che ci avvelena giorno per giorno questa che vorremmo pensare vigilia, e temiamo non sia che una lunghissima notte senza alba. Cerco inutilmente, fino a oggi, qualche

\footnotetext{
${ }^{3}$ Possiamo menzionare l'arte come atto di comunicazione tra lo scrittore e il pubblico, lo scrittore mestierante, rinnovamento della letteratura italiana e del gusto del pubblico ancora legati ai canoni ottocenteschi e alle mode straniere.

${ }^{4}$ Non per caso Bontempelli sceglierà proprio questo articolo come prefazione del volume Avventura novecentista. Selva polemica (1926-1938), la raccolta di articoli che rispecchiano in modo esaustivo, se non addirittura documentaristico, la graduale costruzione della sua poetica.
} 
segno; e più cerco, più mi disoriento. Ci sono state dimostrazioni finite, sfumate tra il silenzio; non n'è uscito un indizio. Alcuni giornali, anche, hanno mutato da un giorno all'altro il loro contegno. A chi si sono venduti? [...] La tensione del nostro spirito è oramai insostenibile, e può essere piena di pericoli nuovi, che nessuno prevede.

O, per meglio dire, si fa portavoce di una delle correnti dell'interventismo che diverge in modo particolare da quella nazionalista, imperialista e futurista. Ce lo conferma l'articolo Momenti e motivi: VII (ottobre 1914) in cui Bontempelli commenta l'impresa del tenente Angelo Belloni, il quale si impossessò di un sommergibile allo scopo di arrivare fino alle coste dell'Albania e attaccare, nonostante la neutralità italiana in quel periodo, la forza navale dell'Impero Austro-Ungarico (Bontempelli 1917: 196-202):

Il "folle volo" del tenente Belloni col suo sottomarino, è quasi un mito nazionale. Sospetto che molti italiani abbiano fatto la propria educazione, per quanto riguarda la guerra e in generale il servizio della patria, più sull'Orlando Furioso che sui libri di storia e su convinzioni di moralità sociale. Temo che molti italiani tendano a considerare la propria partecipazione personale alla guerra più come un'audace $\mathrm{e}$ interessante avventura individuale, che non come un dovere nel quale l'individuo sopprime sé stesso nel sentimento d'una necessità più vasta, trascendente i limiti del suo ardore, della sua vita, della sua gloria.

L'avventura personale in contrasto con le necessità riconosciute della società e del tempo, è il cavaliere in ritardo, è, nel migliore dei casi, Don Chisciotte. [...]

Il Belloni è partito, con modi da romanzo in ottava rima, per compiere qualche cosa di maraviglioso. [...] Si sente già eroe, contemplato, commentato: il suo sommergibile gli pare, a tratti, un palcoscenico: gran bello scenario, per l'eroe sottomarino, l'intero Tirreno!

E il Belloni è indubbiamente un uomo onesto, ardente e geniale. Sì. Lasciatemelo dire: nella educazione politica della genialità italiana, c'è molto Orlando Furioso. E un po' di melodramma metastasiano.

Proprio in quanto rappresentava un momento storico straordinario non solo per l'Italia ma per l'intera Europa, secondo Bontempelli la guerra appena scoppiata richiedeva sobrietà, cautela e senso di responsabilità in luogo di inutili e nocivi atti di eroismo come quello del tenente Belloni. L'educazione politica degli italiani delle ultime generazioni, che poggiava su letture e resoconti anche alquanto favolistici piuttosto che sulle esperienze di guerra vissute, si rispecchiava anche nella loro interpretazione dell'eroe nazionale per eccellenza, Giuseppe Garibaldi: "Troppi italiani si sentono un po' Garibaldi. Ma un Garibaldi a modo loro; non ricordano che la grandezza di lui fu questa appunto, di farsi energico disciplinatore d'energie nativamente scomposte, pericolose per soverchio di personalità. E cominciò dalla propria" (Bontempelli 1917: 198-199). Il nome di Garibaldi, preso 
come incarnazione del genio di improvvisazione e di patriottismo, ricorre di frequente negli scritti interventisti di Bontempelli, come pure in quelli di altri propagatori dell'interventismo democratico, per i quali, come rileva Isnenghi (1984: 537), "Garibaldi può essere uno dei nomi - o magari il più caro e il più grande - della leggenda nazionale della «nuova Italia»: e come tale essere cooptato come garante politico del movimento unitario che giunge a compimento; e, rispetto alle classi popolari, del carattere di liberazione e non di sopraffazione dei popoli assegnato a questa guerra". Sebbene il mito di Garibaldi, come osserva giustamente Di Paola (1984), sia nato già durante la spedizione dei Mille, è con Carducci che Garibaldi diventa "il simbolo della romanità, della forza dell'orgoglio latino, il suscitatore di impeti patriottici" (Di Paola 1984: 519). E Bontempelli, ex carducciano con alle spalle versi classicheggianti ed educazione postrisorgimentale, riprende la "linea garibaldina nella memoria dell'Italiano" (Isnenghi 1984: 539) fino a sfociare, in un'occasione, nello stile oratorio dannunziano, allorquando fa riferimento al "Dio italico" Garibaldi (Bontempelli 1917: 217). Si tratta dell'articolo Momenti e motivi: XI, del 5 maggio 1915, data dell'inaugurazione del Monumento ai Mille di Quarto (avvenuta in concomitanza col cinquantesimo anniversario della partenza dei volontari garibaldini) durante la quale D'Annunzio pronunciò l'Orazione per la Sagra dei Mille (Bontempelli 1917: 217-218):

In questo mezzo secolo l'Italia creata maturò rapidamente il proprio spirito, fino a una nuova stanchezza. E oggi ancora troppe voci consigliano prudenza, e gridano l'Italia immatura a migliori destini. Ma lo spirito di Garibaldi è vivo ancora nel nostro buon popolo audace, maravigliosamente imprudente, provvidamente ignaro delle calme addormentatrici delle diplomazie e delle corti. E oggi il popolo d'Italia giura allo spirito del suo Dio italico, di Garibaldi, di rompere ogni indugio, di rigettare ogni consiglio ambiguo, d'affidarsi al divino istinto ereditato da Lui, e di movere finalmente all'ultimo compimento della Sua impresa. [...]

Sullo scoglio di Quarto, oggi il popolo d'Italia dichiara l'ultima guerra del Risorgimento italiano. E dichiarata la compirà, dovesse la prima battaglia di questa guerra chiamarsi: Rivoluzione.

L'ultimo conflitto del Risorgimento italiano, il carattere rivoluzionario dell'interventismo e la volontà del popolo di entrare in guerra rappresentano solo alcune coordinate dell'interpretazione del fenomeno bellico e delle diverse correnti interventiste che passano anche per le pagine della rivista Lacerba di Papini e Prezzolini, oppure per quelle di Jahier o Agnoletti. L'antiparlamentarismo dei futuristi (Marinetti 1968) o il rifiuto dei partiti espresso da Papini nel 1913 (Isnenghi 1970) costituiscono delle premesse per l'interpretazione della guerra come surrogato della mancata rivoluzione 
antigiolittiana, riscontrabile anche negli articoli di Bontempelli, benché di un tono meno acceso (Bontempelli 1917: 203-204):

Il pubblico è migliore di voi [politici]. Se voglio persuadermi che la nazione è pronta, devo andare in giro, e parlare con quelli che non fanno professione di politica: quanto fervore, quanti riavvicinamenti d'animi e di cervelli che si credevan lontani e di aspirazioni la nuova congiuntura ha creato tra gli italiani; come essi, moltissimi almeno, e d'ogni condizione e d'ogni cultura, sentono la grandezza del momento. Ma se m'accosto ai professionisti della politica e apro i giornali, non sono altrettanto sicuro di quello che sto per trovare.

Bontempelli, dunque, attribuisce abbastanza precocemente l'orientamento interventista al pubblico italiano, ovvero a quei non professionisti di politica che formano l'opinione pubblica già a partire dal novembre 1914, quasi contemporaneamente agli articoli su Lacerba ${ }^{5}$.

Nella primavera del 1915, quando la partecipazione alla guerra sembra sempre più imminente, Bontempelli mostra di condividere con intellettuali di matrice interventista diversa - come Jahier, Prezzolini o Serra - quello che Isnenghi chiama "il movente esistenziale degli interventisti", ovvero la convinzione che, con la perdita dell'occasione della guerra, si perda anche "la speranza d'esser uomo, di sentirsi vivo e giustificato nel proprio esistere" (Isnenghi 1970: 114). A suo parere questo vale in modo particolare per gli intellettuali e gli artisti (Bontempelli 1917: 188-192):

Umiliati e inutili sono e si sentono tutti coloro che [...] non possono, in nessun modo, prender parte attiva alla guerra.

Tra questi, c'è una classe particolarmente infelice: la classe di coloro che hanno per funzione ordinaria, nella società di provvedere, ahimè, ai cosiddetti bisogni dello Spirito: che so io l'arte, la scienza pura, lo studio, il pensiero... Ė tutto un congegno della vita sociale che in una crisi come questa (anche nella primissima crisi, quella dello stupore, del timore e dell'attesa) è il primo ad essere paralizzato, fatto inutile. Di qui la umiliazione, nel senso di una improvvisa inferiorità. [...]

Quanti poeti o filosofi rimpiangono di non saper guidare un aeroplano o un'automobile, o magari umilmente fare il pane e tagliare e cucire le vesti!

L'ultimo articolo della raccolta antecedente l'ingresso in guerra dell'Italia è intitolato Dieci mesi di passione e reca la data del 22 maggio 1915

\footnotetext{
${ }^{5}$ Osserva a proposito Isnenghi (1970: 97) che "Il primo ottobre non si parla più di minoranze, e nell'Appello 'agli italiani intelligenti' è 'il popolo fremente', 'la maggioranza del popolo italiano', che esige ed è pronto a imporre a un governo 'stolto o vile o traditore', secondo una tecnica della diffamazione e dell'oltraggio ad personam che è propria della mobilitazione emotiva che l'interventismo è teso a creare e che in Lacerba e nelle invettive antigiolittiane di D'Annunzio ha i suoi momenti esemplari”.
} 
e offre in modo riassuntivo lo svolgersi della campagna degli interventisti, la cui impazienza sembra raggiungere il suo apice proprio alla vigilia dell'entrata nel conflitto (Bontempelli 1917: 43-45):

Il manifesto verde della mobilitazione su per i muri delle città - l'annuncio ufficiale della dichiarazione di guerra - (o noi a loro, o loro a noi, poco importa!), - le notizie dei primi sconfinamenti, delle prime battaglie - l'eco del cannone e l'odor della polvere, che dalle frontiere parrà diffondersi per tutta l'aria della penisola: siamo impazienti di tutto questo. Ci occorre una notizia nuova ogni giorno; qualche cosa di più, ogni giorno; il crescendo, fino allo scoppio. I nostri nervi sono esasperati. Troppi contorcimenti hanno subito già, per poter sopportare altre ore di silenzio. [...] Un ardore che ha potuto mantenersi, accrescersi, infervorarsi, dilagare sempre più fra tanta vicenda di entusiasmi e di abbattimenti, è una energia formidabile. Ma sarebbe pericoloso protrarne lo scoppio. Ogni cittadino italiano è nella condizione di un soldato appiattato in trincea che aspetta l'ordine.

Nella sua analisi dell'interventismo, Bontempelli (1917: 46) non solo sostiene l'estraneità e l'indifferenza degli italiani nei confronti della Triplice Alleanza ("Non se ne conoscevano né la sostanza né la portata. Ci appariva come una formula vaga, astratta, metafisica. Era lontana dal nostro sentimento e dal nostro interessamento"), ma attribuisce - e a ragione - alla stampa e ai giornali un ruolo fondamentale nella nascita e nell'evolversi della causa interventista: "I giornali si costituirono in cattedre di storia, d'economia e di politica; e l'intuito magnifico del popolo italiano ne assimilò rapidamente gli insegnamenti. La prima intuizione e le nuove conoscenze confluirono, e nacque l'interventismo" (Bontempelli 1917: 56). Costituendo il primo mezzo vero e proprio di comunicazione di massa, i giornali (in)formano l'opinione pubblica e, attraverso i loro redattori, ridefiniscono il ruolo dell'intellettuale in tempo di guerra. Non a caso, dunque, saranno mandati al fronte numerosi inviati speciali, tra cui, si diceva in apertura, anche Bontempelli.

Un'altra caratteristica del discorso interventista che lo scrittore comasco adotterà a partire da questo articolo è poi relativa alla retorica antigermanica. Così, una delle tre fonti del neutralismo in Italia, accanto all'ideologia e alla vigliaccheria, è la germanofilia (Bontempelli 1917: 61-62):

La germanofilia. Troppi esempi l'hanno illustrata, perché occorra ricordarne i modi e gli effetti. La burocrazia universitaria, l'indirizzo della educazione tecnica militare, gli interessi economici (da quelli maggiori delle industrie e delle Banche italiane che vivevano di capitali tedeschi, e Milano ne sa qualche cosa, a quelli minimi degli affittacamere con clientela tedesca e dei mariti con mogli tedesche ben dotate), la devozione supina del debole per la forza fisica pura - crearono una corrente torbida di apparente ammirazione per la potenza germanica. 
Dopo 1'entrata dell'Italia in guerra, Bontempelli, nelle vesti di corrispondente di guerra, non solo non abbandona le idee interventiste, ma le riconferma e rielabora, affinandole ulteriormente e cercando di giustificare la scelta dell'Italia. Nell'articolo Gli uomini e $i$ tedeschi (giugno 1915) la retorica antigermanica incontra quella garibaldina, postrisorgimentale in quanto la prima guerra mondiale viene considerata una 'impresa italiana di indipendenza' che fa contrapporre da una parte il genio militare italiano rappresentato da Garibaldi e dall'altra le modernissime armi di guerra di produzione tedesca (Bontempelli 1917: 78-79):

Ed è inoltre da considerare che l'ammirazione deve nascere piuttosto verso il modo di servirsi di uno strumento, che non verso il fatto di possederlo: penso che anche militarmente un'azione di Garibaldi, compiuta sempre contro milizie più agguerrite, con soldati improvvisati, con bande impreparate, con organismi estremamente semplici, ma avvivati dalla fiamma inimitabile del genio - genio suo personale, e genio di razza - sia le mille volte più meravigliosa che le mastodontiche carneficine compiute dalla Germania d'oggi, con istrumenti perfezionati in una lunghissima preparazione, contro nemici già anch'essi occultamente indeboliti in lunghe e perverse opere di tradimento, durante gli anni che tutti credevano di pace.

Appena iniziata la guerra, quindi, Bontempelli cerca in un certo senso di smitizzare quei luoghi comuni che gli italiani hanno alimentato nei confronti dei tedeschi e di alzare così il morale sia dei civili che dei primi soldati mandati al fronte. E un luogo comune ben radicato nelle ultime generazioni degli italiani è indubbiamente quello che vede il tedesco come l'uomo di scienza e di pensiero per eccellenza (Bontempelli 1917: 81-82):

Il borghese, il provinciale della cultura, si lascia imporre soprattutto dal titolo, dalla consacrazione accademica. Non ha altro modo di controllo. Per lui il culmine della intelligenza è rappresentato dal professore d'Università. Il tedesco è il professore d'Università per eccellenza. Ora, l'Università è tutt'altro che la regione privilegiata dell'intelligenza, tutt'altro che il centro di elaborazione dell'energia spirituale dell'uomo. Ne è, nel migliore dei casi, l'archivio, lo schedario: spesso la caricatura. L'effetto dell'educazione universitaria su di una generazione giovane che le sia affidata nel momento più fattivo del proprio sviluppo, è deleterio. Solo le più forti energie vi sfuggono; le altre ne escono deformate, soffocate. L'Università è mediocrificatrice per eccellenza. E il tedesco, quando non è soldato, è professore, professore di Università, per eccellenza.

Analogamente ai futuristi, anche Bontempelli cerca di porre fine all'ammirazione per la Germania (e l'Austria) intellettuale, per il "professorume" e i "tedescofili", opponendo il genio italiano e la capacità di improvvisazione all'eccessiva organizzazione, al rigore e intellettualismo della civiltà germanica: "Uno dei benefici spirituali del decadimento germanico in Europa, 
sarà appunto di liberarcene. Abbiamo bisogno di freschezza, di giovinezza, e, sì, di improvvisazione: sono le qualità nostre, e non sono affatto contrarie alla serietà, come molti credono ancora" (Bontempelli 1917: 84). A nostro parere, quello che risulta particolarmente interessante nei suoi scritti dei primi mesi della guerra, è che Bontempelli, nonostante continui a fare propaganda probellica, riafferma le sue posizioni contro la cultura tedesca (Bontempelli 1917: 86-87):

Qualcuno può credere che l'antigermanesimo della propaganda fatta sino a ieri fosse appunto una esagerazione propagandistica, un mezzo per preparare la guerra speciale contro l'Austria; e può desiderare che, vinta questa, si debba ritornare a un'equa visione dei meriti della Germania, a un riconoscimento dei suoi benefici, ecc. Ciò va impedito. Sarebbe deleterio. [...]

L'odio contro il tedesco va mantenuto vivo. È necessario ricordar sempre, fin da ora, che la guerra italiana di nazionalità è parte - e quale parte! - di una guerra di civiltà, da cui il germanesimo dei mutilatori di bimbi deve uscire distrutto.

Non dobbiamo dimenticare i bimbi mutilati del Belgio. Sono il più chiaro segno dell'animo della Germania e della sua funzione in Europa.

Bontempelli assumerà lo stesso atteggiamento antitedesco anche nell'affrontare un altro motivo ricorrente della propaganda interventista di stampo democratico, cioè quello della Grande guerra intesa come l'ultima guerra d'indipendenza che traeva le sue origini dal Risorgimento (Bontempelli 1917: 118-120):

Per tutte queste ragioni (che accenno soltanto e potrebbero lungamente svolgersi e dimostrarsi), per levare in armi l'Italia dovemmo darle una guerra che si ricollegasse strettamente alla sua più vicina, gloriosa e popolare tradizione storica, che non fosse se non la continuazione di una gesta cui il padre di ognuno di noi aveva partecipato, il cui ardore aveva riscaldato gli animi di ognuno di noi fin dagli anni in cui cominciavamo a imparare a leggere sui libri in tutti $i$ quali era l'immagine di Garibaldi.

All'Italia si dovè dare la quarta guerra d'indipendenza contro l'Austria e per mezzo di questa porla a fianco delle nazioni che la Germania minacciava apertamente, mentre alla nostra preparava la rovina con mezzi obliqui e occulti.

L'impostazione - diplomatica, militare e politica - della guerra d'Italia come guerra di indipendenza contro l'Austria, contribuì ad accrescere tra noi il senso di diversità di fini, di molteplicità di guerre, e farci perdere la veduta iniziale di una levata difensiva di tutte le nazioni civili contro le minacce della grossa barbarie tedesca.

Benché egli ammetta che il concetto di quarta guerra d'indipendenza contro l'Austria sia una costruzione diplomatica, militare e politica, Bontempelli non rinuncia a quel collegamento al Risorgimento, riprendendo le battaglie e i nemici dei padri e dei nonni dei suoi coetanei (Bontempelli 1917: 132-133): 
Nella grande maggioranza delle famiglie del lombardo-veneto c'è stato un padre che giovane ha combattuto con Garibaldi e un nonno che ha passato il più della vita sotto il regime austriaco. Nella primissima fanciullezza di coloro che oggi sono intorno ai trent'anni, l'austriaco appare come il nemico naturale ed ereditario della razza: questo specialmente tra il popolo, che è miglior conservatore dei sentimenti trasmessi per tradizione.

Una simile ingenuità, o per dirla in termini bontempelliani, un certo candore, unitamente a convinzioni nazionaliste e a sentimenti patriottici, risulta in modo particolarmente evidente dalle meditazioni sulle ragioni della partecipazione dell'Italia alla Grande guerra. Nell'articolo Tendenze, appartenente allo stesso periodo dei primi mesi della guerra, Bontempelli (1917: 233) parte dalla convinzione comune che "una volontà imperialistica dell'Italia, $[\ldots]$ nascosta o soffocata dietro le apparenze o sotto la compressione della sua vita parlamentare, vagamente democratica, preparò in ogni modo, all'infuori delle sfere ufficiali l'Italia del 1915 e di domani, e [che] trovò nella guerra un'inaspettata e felice occasione di attuare la propria energia lungamente maturata e complessa". In effetti, questa convinzione viene confermata anche da Isnenghi (1970: 29): "I moventi 'nazionali', esplicitamente e direttamente politici - e in particolare i moventi liberaldemocratici - arrivano tra gli ultimi. Il terreno, per anni, era stato preparato all'interventismo dall'indissolubile intreccio dei moventi psicologico-esistenziali d'impronta vitalista e di quelli politici d'impronta imperialista, volti, insieme, alla sovversione interna dell'assetto democratico-liberale". Bontempelli, fedele alle convinzioni maturate durante la campagna interventista, attribuisce però la partecipazione dell'Italia alla guerra innanzitutto a ragioni storiche e culturali, ricorrendo al discorso dell'antagonismo tra le civiltà europee, particolarmente tra quella tedesca e italiana (Bontempelli 1917: 237-238):

Smania di imparare e imparar presto ogni cosa, da chiunque potesse insegnarcela, fu quella che ci mise nelle mani dei tedeschi, in modo che la scossa per strapparcene dovè e deve essere d'una violenza di cui noi stessi ci credevamo incapaci. Tanto poco eravamo imperialisti, che non chiedevamo neppure di fare da noi quello che avremmo potuto, ma pareva che ci studiassimo di portarci in casa in tutti i modi il nemico, per impararne le industrie e raggiungere più presto i beneficii, al tutto materiali, della sua esperienza, senza menomamente diffidare dei possibili effetti di tale sottomissione. La quale - tanto poco era il nostro orgoglio - passò presto dagli ordini materiali della vita agli intellettuali: e finimmo proprio all'opposto del punto di partenza, finimmo col prender ogni cosa da qualcuno. E non dai tedeschi soli. Pigliavamo, per esempio, il teatro dalla Francia e il romanzo dai nordici e la moda del vestire di Francia e d'Inghilterra, come di Germania le macchine e l'educazione universitaria. 
Dunque, un rinnovamento totale dei vari aspetti della giovane nazione italiana fu, a suo avviso, il motore principale dell'opposizione all'egemonia industriale e culturale della Germania, e ciò non coincideva in modo assoluto (o, addirittura, nemmeno in minima parte) con quanto sostenuto dalla tendenza imperialista (Bontempelli 1917: 234-235):

La formula della sua volontà intima era «crearsi una nuova anima», degna dell'antica, degna della nuova situazione nel sistema dei popoli, degna dell'avvenire.

Tale volontà fu di rinnovamento intimo, di consolidamento nel profondo, di perfezionamento di tutte le proprie facoltà, pratiche e spirituali, industriali, commerciali, intellettuali, morali; ma in questo desiderio di perfezionamento non entrava che in menoma parte (e troppo piccola in verità, credo), e forse anzi non entrava affatto, la tedenza espansionistica, egemonica, imperialista, in nessuna delle sue forme.

Idealizzando motivazioni probelliche di quella parte della nazione che Bontempelli chiama "popolo (italiano)", "tendenza popolare", "corrente popolare" o "l'Italia popolare", egli ancora una volta sostiene due aspetti fondamentali della Grande guerra per quanto riguarda l'Italia, cioè quelli di "guerra d'indipendenza" e "guerra cavalleresca" (Bontempelli 1917: 241-242):

Come guerra d'indipendenza, si ricollega direttamente alla idealità che fin dall'adolescenza ci fece amare Garibaldi e la Storia del Risorgimento, ed è, come fu il Risorgimento, un atto insieme di sentimento e di cultura. Come guerra cavalleresca, è il risultato della ribellione contro l'inumanità, l'antistoricità, la medievalità dell'attentato tedesco; e la pietà per il Belgio e la Serbia, e, nei primi mesi, il terrore per la sorte della Francia, operarono come motivi principalissimi nella nostra veduta e nel nostro entusiasmo.

È da notare in questi passaggi che l'italianità di stampo tradizionale, che attinge ai valori e alle idee risorgimentali e che talvolta vagheggia anche il passato romano, è ben diversa dal modello nazionalista ed imperialista propagato, per esempio, dai futuristi. Anche Bontempelli, in effetti, distingue tra la corrente popolare e quella nazionalista o imperialista, le quali, nel momento opportuno, si unirono per combattere insieme lo stesso nemico (Bontempelli 1917: 243):

L'idealismo della nostra tendenza popolare, e il realismo della tendenza nazionalistica scopersero, allo scoppiare della guerra, una improvvisa e insospettata coincidenza d'interessi. E la grande corrente popolare - umanitaria e spiritualistica di sua natura - e la piccola corrente nazionalista o imperialista - d'atteggiamento eminentemente pratico e realistico - si mescolarono in questa coincidenza, e si trovarono a collaborare alla grande impresa, senza che l'una d'esse possa vantarsi d'aver sopraffatto o assorbita l'altra. 
Bontempelli, quindi, nega la ricerca di supremazia come fine della guerra, che era una posizione della tendenza imperialista. Pur riconoscendone un ruolo nello spingere il popolo italiano in guerra, ripropone motivi superiori e idealistici (Bontempelli 1917: 260-261):

Concludendo: io non nego, anzi affermo e desidero, che la nostra sia guerra nazionale da cui l'Italia debba uscire, oltre che territorialmente integrata, maggiore in ogni senso e più forte. Ma nego che questo sforzo debba essere di natura sfrenatamente egemonica: nego che non possa esservi qualche forza superiore, di natura ideale, che limiti le possibilità imperialistiche dei popoli; rifiuto la negazione nazionalistica di vantaggi ideali, nel senso umanitario, che la vita della storia possa conquistare al divenire del mondo; respingo similmente la negazione di superiori simpatie e ragioni ideali che debbano aiutare le nazioni in certe conquiste comuni, e nel tempo della guerra e in quello della pace, e cementarle tra loro più durevolmente che in una alleanza militare.

In conclusione, si può sostenere che l'attività giornalistica del comasco durante la campagna interventista riconfermi ciò che era evidente sin dal lancio del movimento futurista del 1909, e cioè che Bontempelli e Marinetti optarono per due ipotesi di modernità diverse (Benussi 2009), come si evince anche dalla differente retorica interventista. Bontempelli, a trentasei anni compiuti, critico, redattore, giornalista e autore di poesie e racconti che rispettavano ancora $\mathrm{i}$ canoni tradizionali, è un interventista sobrio e prudente, un intellettuale e patriota che auspica una ripresa dei valori risorgimentali; ed è questo che, propriamente, lo accomuna agli interventisti di matrice democratica. Benché la guerra rappresenti per entrambi un evento straordinario che dovrebbe rinnovare la vita della ancora giovane nazione italiana in tutti i suoi aspetti, la concezione della guerra come il più bel poema futurista apparso finora, nonché fonte di ispirazione artistica, è ancora lontano nelle prime meditazioni bontempelliane sulla Grande guerra. Bontempelli, infatti, diffida delle imprese eroiche e superomistiche di un Belloni, richiamando piuttosto alla prudenza e all'autocontrollo ammirati nell'eroe del Risorgimento Giuseppe Garibaldi. E se, da una parte, Marinetti distingue tra due concezioni opposte di guerra, quella passatista e futurista, Bontempelli sottolinea, come abbiamo visto, la differenza tra due correnti interventiste, quella dell'Italia popolare e quella imperialista. I due "ultimi mitografi del Novecento" (Tondelli 2009) si incontrano nell'uso di una retorica antigermanica con la differenza che Marinetti accosta alla cultura germanica anche quella latina poiché "tutte e due queste culture [sono] ugualmente nocive, [vanno combattute] per difendere il genio creatore italiano d'oggi" (Marinetti 1968: 289). Per il fondatore del futurismo, infatti, la cultura latina è strettamente legata a quella ottocentesca, carducciana e quindi passatista, ma è proprio a tale cultura che Massimo Bontempelli 
nel periodo dell'interventismo e nei primi anni della Grande guerra ancora inevitabilmente appartiene. Ed è tuttavia interessante ravvisare che, dopo il trasferimento a Milano, dopo i primi incontri con gli esponenti del futurismo e, soprattutto, dopo un contatto diretto con il fronte da inviato speciale, Bontempelli finirà per includere nella sua concezione della guerra quella valenza vitalistica e bellicista tipica della retorica futurista. In virtù di ciò, il loro rispettivo schieramento interventista non esprime solo delle divergenze, ma costituisce anche le fondamenta di quello che diventerà un breve ma intenso sodalizio a sua volta cruciale per il successivo sviluppo della poetica bontempelliana.

\section{BIBLIOGRAFIA}

Benussi, C. (2009). Lucini e Marinetti: linguaggi, figure, miti. Due ipotesi di modernità. In G. E. Viola (a cura di), Una bellezza nuova (pp. 211-225). Roma: Biblioteca d'Orfeo.

Bontempelli, M. (1915). Dallo Stelvio al mare. Firenze: R. Bemporad \& figlio.

Bontempelli, M. (1917). Meditazioni intorno alla guerra d'Italia e d'Europa. Milano: Istituto Editoriale Italiano.

Bontempelli, M. (1928). Il neosofista e altri scritti (1920-1922). Milano: Mondadori.

Bontempelli, M. (1938). L'avventura novecentista: selva polemica, 19261938: dal realismo magico allo stile naturale, soglia della terza epoca. Firenze: Vallecchi.

Di Paola, S. (1984). Il mito di Garibaldi nella poesia italiana. In F. Mazzonis (a cura di), Garibaldi condottiero. Storia, teoria, prassi. (pp. 507-521). Milano: Franco Angeli.

Isnenghi, M. (1970). Il mito della grande guerra: da Marinetti a Malaparte. Bari: Laterza.

Isnenghi, M. (1984). Usi politici di Garibaldi dall'interventismo al fascismo. In F. Mazzonis (a cura di), Garibaldi condottiero. Storia, teoria, prassi (pp. 533-544). Milano: Franco Angeli.

Marinetti, F. T. (1968). In quest'anno futurista. In L. De Maria (a cura di), Teoria e invenzione futurista/F. T. Marinetti (pp. 130-135). Milano: Mondadori.

Papini, M. C. (a cura di) (1977). L'Italia Futurista (1916-1918). Roma: Edizioni dell'Ateneo\&Bizzarri.

Tempesti, F. (1974). Massimo Bontempelli. Firenze: La Nuova Italia.

Tondelli, L. (2009). Futurista senza futuro. Marinetti ultimo mitografo. Firenze: Le Lettere. 


\title{
MASSIMO BONTEMPELLI'S INTELECTUAL INTERVENTIONISM BETWEEN JOURNALISM AND FUTURIST BELLICISM
}

\begin{abstract}
Summary
This article aims to illustrate the development and characteristics of Massimo Bontempelli's interventionism by examining his newspaper articles and putting emphasis on possible points of convergence and differences when compared to futurist interventionism. His interventionist articles published in the volume Meditazioni intorno alla guerra d'Italia e d'Europa [Meditations on War of Italy and Europe] in 1917 reveal, in fact, that Bontempelli's interventionism can be put into relation with a democratically oriented interventionism rather than nationalistic and imperialistic currents due to its retrieving of the Risorgimento's values, political use of the figure of Giuseppe Garibaldi and antigermanic rhetorics. In spite of the intial ideological divergences with the Futurist interventionist discourse, it is actually the intervention campaign that makes Bontempelli and Marinetti's movement find common ground first politically and later on artistically as well.

Keywords: Bontempelli, interventionism, journalism, the Great War, Futurism, bellicism.
\end{abstract}

